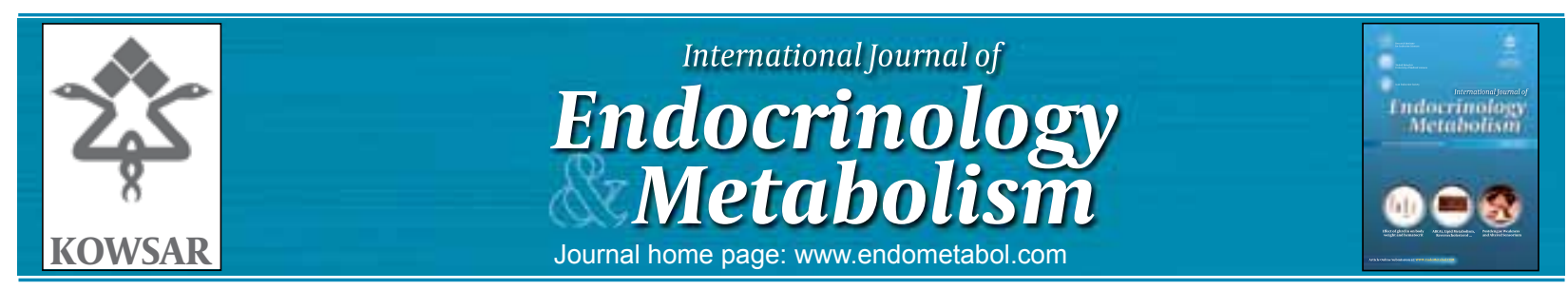

\title{
The Sesame Lignan Sesamin Attenuates Vascular Permeability in Rats with Streptozotocin-Induced Diabetes: Involvement of Oxidative Stress
}

\author{
Mehrdad Roghani $^{1^{*}}$, Tourandokht Baluchnejadmojarad ${ }^{2}$, Farshad Roghani Dehkordi ${ }^{3}$ \\ ${ }^{1}$ Department of Physiology, School of Medicine and Medicinal Plant Research Center, Shahed University, Tehran, IR Iran \\ ${ }^{2}$ Department of Physiology, School of Medicine, Tehran University of Medical Sciences, Tehran, IR Iran \\ ${ }^{3}$ Department of Cardiology and Internal Medicine, School of Medicine, Isfahan University of Medical Sciences, Tehran, IR Iran
}

\section{A R T I C L E I N F O}

Article Type:

Original Article

Article history:

Received: 02 Dec 2010

Revised: 10 Dec 2010

Accepted: 02 Jan 2011

\section{Keywords:}

Sesamin

Sesame

Diabetes mellitus

Capillary permeability

Oxidative stress

\section{A B S T R A C T}

Background: Cardiovascular disorders are a major cause of morbidity and mortality in diabetic patients. Increased vascular permeability is a hallmark of diabetic vasculopathy, and the administration of natural products with antioxidant activity could restore vascular function.

Objectives: In this study, the effect of chronic treatment with sesamin on vascular permeability in rats with streptozotocin (STZ)-induced diabetes was investigated.

Materials and Methods: Male diabetic rats received sesamin at a dose of either 10 or 20 $\mathrm{mg} / \mathrm{kg}$ for 7 weeks, beginning 1 week after diabetes induction. Vascular permeability was estimated by measuring Evans blue dye extravasation. Oxidative stress markers, including malondialdehyde (MDA) and superoxide dismutase (SOD) activity, were also measured in aortic tissue.

Results: Extravasation of Evans blue dye increased significantly in the diabetic group compared to that in the control group $(p<0.05)$, and treatment with sesamin significantly and dose-dependently decreased this extravasation $(p<0.05)$. Diabetic rats also had elevated malondialdehyde (MDA) and reduced superoxide dismutase (SOD) activity $(p<0.005-0.001)$, and chronic treatment with sesamin $(20 \mathrm{mg} / \mathrm{kg})$ significantly reversed the elevated MDA content $(p<0.05)$ and reduced SOD activity $(p<0.05)$.

Conclusions: Chronic treatment of diabetic rats with sesamin could dose-dependently improve aortic permeability, partly through the attenuation of oxidative stress in aortic tissue.

๑ 2011 Kowsar M.P.Co. All rights reserved.

\footnotetext{
- Implication for health policy/practice/research/medical education:

This work may pave the way for designing new treatments for attenuation of some diabetic complications due to increased vascular permeability.
}

\footnotetext{
- Please cite this paper as:

Roghani M, Baluchnejadmojarad T, Roghani Dehkordi F. The Sesame Lignan Sesamin Attenuates Vascular Permeability in Rats with Streptozotocin-Induced diabetes: Involvement of Oxidative Stress. Int J Endocrinol Metab. 2011; 9(1): 248-52. DOI: 10.5812/ Kowsar.1726913X.2142
}

\section{Background}

Diabetes mellitus (DM) is a major health problem in the 21st century; the prevalence of DM is increasing worldwide, and it is estimated to effect 366 million people by

\footnotetext{
* Corresponding author at: Mehrdad Roghani, Department of Physiology, School of Medicine, Shahed University, Tehran, IR Iran. Tel: +982188964792, Fax: +98-2188966310,E-mail: mehjour@yahoo.com DOI: 10.5812/Kowsar.1726913X.2142

Copyright $\odot 2011$, Iran Endocrine Society, Published by Kowsar M.P.Co. All rights reserved.
}

the year 2030 (1). Cardiovascular disorders continue to be the main cause of morbidity and mortality in diabetic patients, despite significant achievements in their diagnosis and treatment (2). Diffuse vasculopathy is a common feature of type 1 diabetes, and is characterized by increased vascular permeability and subsequent plasma extravasation (3). Most vascular complications in diabetes are due to increased serum glucose and augmented oxidative stress (4). 
Sesamin, a major lignan in sesame seeds and oil, and its isomers have beneficial physiological effects, including antioxidant (5), anti-carcinogen (6), and anti-hypertensive activities $(7,8)$, and are capable of reducing serum lipids (9). Sesamin isomers have been suggested to enhance plasma levels of $\alpha$ - and $\gamma$-tocopherol in rats (10). Furthermore, recent studies have demonstrated that sesamin metabolites induce nitric oxide-dependent vasorelaxation in vitro (11), and that sesamin feeding enhances endothelium-dependent relaxation in deoxycorticosterone acetate (DOCA)-salt hypertensive rats (8).

\section{Objectives}

An aqueous extract of leaves from the sesame plant was previously shown to induce dose-dependent vasorelaxation in guinea pig aortas (12). However, the in vivo protective effect of sesamin against the vascular permeability in diabetes has not yet been documented. Therefore, this study was designed to assess, for the first time, the beneficial effect of chronic treatment with sesamin on the improvement of vascular permeability in rats with streptozotocin (STZ)-induced diabetes and to investigate the possible involvement of oxidative stress.

\section{Materials and Methods}

\subsection{Animals}

Male albino Wistar rats (Pasteur institute, Tehran, Iran) weighing 240-300 g were housed in an air-conditioned colony room at $19-23^{\circ} \mathrm{C}$ and were supplied with a standard pellet diet and tap water ad libitum. Procedures involving animals and their care were conducted in conformity with NIH guidelines for the care and use of laboratory animals (NIH publication 86-23, revised 1985).

\subsection{Chemicals}

Streptozotocin, formamide, sesamin, and components for SOD and MDA kits were purchased from Sigma Chemical (St. Louis, MO, USA). All other chemicals were purchased from Merck (Germany).

\subsection{Experimental Protocol}

The rats were rendered diabetic by a single intraperitoneal dose of $60 \mathrm{mg} \cdot \mathrm{kg}^{-1}$ of STZ that was freshly dissolved in ice-cold $0.1 \mathrm{M}$ citrate buffer ( $\mathrm{pH} 4.5$ ). Age-matched normal animals that received an injection of an equivalent volume of buffer comprised a non-diabetic control group. One week after STZ injection, overnight fasting blood samples were collected and serum glucose concentrations were measured using the glucose oxidation method (Zistchimie, Tehran). Only those animals with a serum glucose level higher than $250 \mathrm{mg} / \mathrm{dL}$ were selected as diabetic. During the following weeks, diabetes was reconfirmed by the presence of polyphagia, polydipsia, polyuria, and weight loss. Normal and hyperglycemic rats (a total of 48) were randomly allocated and placed into 6 groups (3 per group): normal vehicle-treated control, sesamin-treated controls (2 subgroups), diabetic, and sesamin-treated diabetics (2 subgroups). Sesamin dissolved in carboxymethylcellulose was administered p.o. (using a gavage needle) at a dose of either 10 or 20 $\mathrm{mg} / \mathrm{kg}$ b.w. daily throughout the 7-week experimental period. Changes in body weight were recorded regularly during the study.

\subsection{Measurement of Vascular Permeability}

The Evans Blue (EB) dye extravasation technique was used to measure the permeability to albumin of capillaries in the aortic tissue from anesthetized rats. This technique is based on the principle that EB dye avidly binds to intravascular albumin, and is thus a reliable way to assess transvascular fluxes of macromolecules. This technique has been extensively validated and has been shown to be a reliable estimate of the extravasation and interstitial accumulation of albumin as previously described (13). Briefly, rats were anesthetized with a combination of ketamine $(100 \mathrm{mg} / \mathrm{kg})$ and xylazine $(8 \mathrm{mg} / \mathrm{kg})$, and then given an injection of EB dye saline solution $(20 \mathrm{mg} / \mathrm{kg})$ in the femoral vein. The dye was allowed to circulate for $10 \mathrm{~min}$. Then, in order to remove any intravascular dye that would interfere with the EB that extravasated in aortic tissue, the thorax was cut and a transcardial perfusion with $100 \mathrm{~mL}$ of heparinized saline was applied through the left ventricle. Next, the descending aorta was dissected out and immediately weighed. One-third of each tissue sample was dried at $60^{\circ} \mathrm{C}$ for 24 hours and a dryl wet weight ratio was calculated. The remaining twothirds of each sample was placed in formamide solution ( $2 \mathrm{~mL} / 200 \mathrm{mg}$ wet tissue) at $25^{\circ} \mathrm{C}$ for 24 hours to extract the dye. The amount of EB dye extracted was determined spectrophotometrically at $620 \mathrm{~nm}$. The results were calculated from an EB dye standard curve (0.5-25 mg/mL), and was expressed as $\mu \mathrm{g}$ of EB dye per $100 \mathrm{mg}$ of tissue dry weight.

\subsection{Determination of MDA Concentration in Aortic Rings}

After removing aortic segments and cleansing to remove extra tissue, they were blotted dry, weighed, and then processed to make a $5 \%$ tissue homogenate in an icecold $0.9 \%$ saline solution. The supernatant of the tissue homogenate was obtained by centrifugation at 1,000 $\times g$ for $5 \mathrm{~min}$ at $4^{\circ} \mathrm{C}$. The MDA concentration (thiobarbituric acid reactive substances, TBARS) in the supernatant was measured as described previously (14). Briefly, trichloroacetic acid and TBA solutions were added to the supernatant, which was then mixed and incubated at $100^{\circ} \mathrm{C}$ for $80 \mathrm{~min}$. After cooling on ice, the samples were centrifuged at $1,000 \times g$ for $20 \mathrm{~min}$, and the absorbance of the supernatant was read at $532 \mathrm{~nm}$. TBARS results were expressed as MDA equivalents using tetraethoxypropane as a standard. 
Table 1. Malondialdehyde (MDA) Content and Superoxide Dismutase(SOD) Activity in the Aortic Tissue of Diabetic Rats with or without Sesamin Treatment

\begin{tabular}{llll}
\hline & n & MDA, $\mu \mathbf{m o l} / \mathbf{g}$ Protein & SOD Activity, kNU/g protein \\
\hline Control & 7 & $5.7 \pm 0.5$ & $117 \pm 6$ \\
Control + Sesamin 20 & 6 & $5.5 \pm 0.4$ & $119 \pm 7$ \\
Diabetic & 6 & $9.2 \pm 0.7^{\mathrm{b}}$ & $76 \pm 8^{\mathrm{a}}$ \\
Diabetic + Sesamin 20 & 6 & $6.9 \pm 0.5^{\mathrm{c}}$ & $105 \pm 8^{\mathrm{c}}$ \\
\hline
\end{tabular}

Abbreviation: kNU, Kilo Nitrite Unit

${ }^{\mathrm{a}} \mathrm{p}<0.005$

${ }^{\mathrm{b}} p<0.001$ (vs. the control group)

${ }^{c} p<0.05$ (vs. the diabetic group)

\subsection{Measurement of SOD Activity in Aortic Rings}

The tissue homogenate supernatant was obtained and SOD activity was measured as described previously (15). Briefly, the supernatant was incubated with xanthine and xanthine oxidase in potassium phosphate buffer ( $\mathrm{pH} 7.8)$ at $37^{\circ} \mathrm{C}$ for $40 \mathrm{~min}$. NBT was added, and blue formazan formation was monitored spectrophotometrically at 550 $\mathrm{nm}$. The amount of protein that inhibited NBT reduction to $50 \%$ of maximum was defined as 1 nitrite unit (NU) of SOD activity.

\subsection{Protein Assay}

The protein content of the supernatant was measured by the Bradford method using bovine serum albumin (Sigma Chemical) as the standard (16).

\subsection{Data and Statistical Analysis}

All values are presented as mean \pm SEM. Statistical analyses were carried out using repeated measure ANOVA and one-way ANOVA followed by Tukey post-hoc test. A p value of less than 0.05 was considered statistically significant.

\section{Results}

After the 8-week experimental period, the weight of the vehicle-treated diabetic rats was significantly lower than

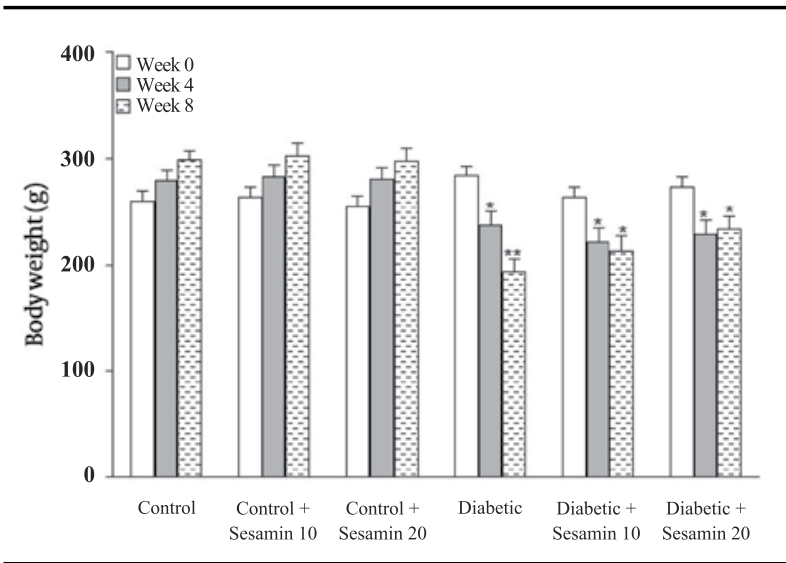

Figure 1. Body Weight of Rats 1 Week before Diabetes Induction and at 4 and 8 weeks after Induction

The data shown are mean \pm SEM. Sesamin10 and sesamin 20 stand for sesamin at doses of 10 and $20 \mathrm{mg} / \mathrm{kg}$, respectively.

${ }^{*} p<0.05 ;{ }^{* *} p<0.005$ (compared to week 0 in the same group) that of the controls $(p<0.005)$, and sesamin treatment at both doses, but particularly at $20 \mathrm{mg} / \mathrm{kg}$, caused a nonsignificant increase in the weight of diabetic rats compared to vehicle-treated diabetic rats (Figure.1). Untreated diabetic rats also had elevated serum glucose levels compared to those of control rats $(p<0.0005)$, and treatment with sesamin, especially at a dose of $20 \mathrm{mg} / \mathrm{kg}$, caused a non-significant decrease in serum glucose compared to

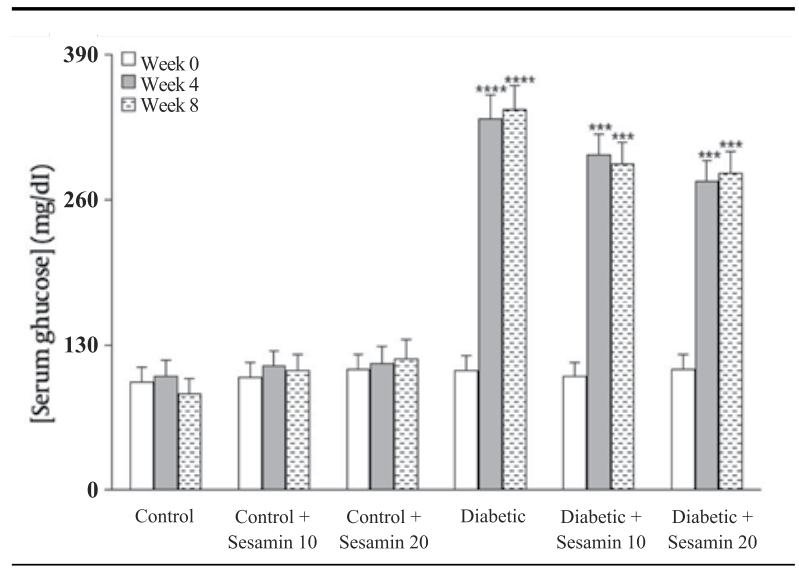

Figure 2. Serum Glucose Concentration 1 Week before Diabetes Induction and at 4 and 8 Weeks after Induction

The data shown are mean \pm SEM. Sesamin 10 and sesamin 20 stand for sesamin at doses of 10 and $20 \mathrm{mg} / \mathrm{kg}$, respectively

${ }^{* * *} p<0.001 ;{ }^{* * * *} p<0.0005$ (compared to week 0 in the same group).

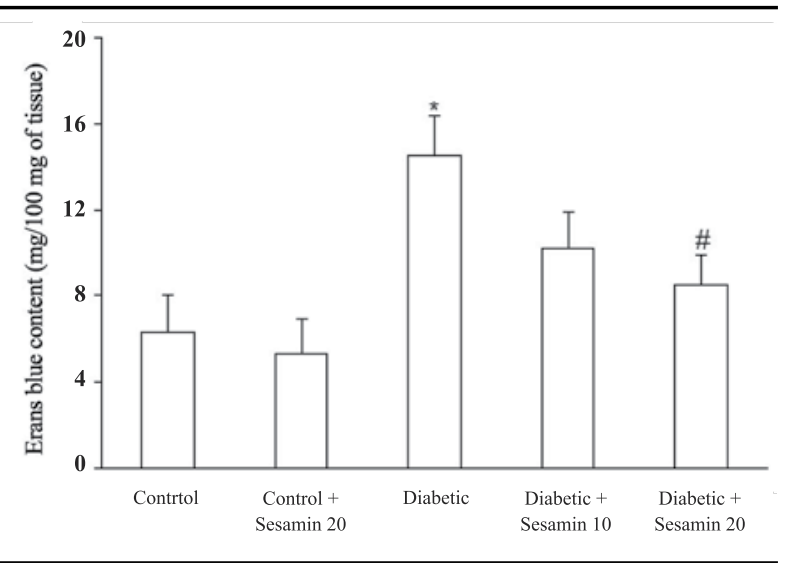

Figure 3. Permeability of Aortic Tissue Measured by Extravasation of Evans Blue Dye ( $\mu \mathrm{g} / 100 \mathrm{mg}$ Tissue) in Different Groups

Sesamin 10 and sesamin 20 stand for sesamin at doses of 10 and $20 \mathrm{mg} / \mathrm{kg}$, respectively.

${ }^{*} p<0.05$ (vs. the control group); $\#<<0.05$ (vs. the diabetic group) 
the diabetic rats. In addition, sesamin treatment of control rats did not produce any significant change in serum glucose levels (Figure. 2). As a measurement of aortic permeability, extravasation of Evans blue dye from the capillaries of rats in the diabetic group increased significantly (by $130.2 \%$ ) than that in the rats in the control group $(p<$ 0.05 ), and treatment of the diabetic group with $20 \mathrm{mg}$ / $\mathrm{kg}$ sesamin significantly decreased this extravasation $(p$ $<0.05$ ). There was no significant difference in extravasation between the sesamin-treated and vehicle-treated control groups (Figure. 3). Measurement of aortic lipid peroxidation markers (Table 1) showed that STZ-induced diabetes resulted in elevated MDA content and reduced SOD activity in aortic tissue $(p<0.005-0.001)$, and chronic treatment of the diabetic group with sesamin $(20 \mathrm{mg} /$ $\mathrm{kg}$ ) significantly reversed the elevated MDA content and reduced SOD activity $(p<0.05)$.

\section{Discussion}

In this study, administration of sesamin for 7 weeks did not have a significant hypoglycemic effect; however, it did reduce the enhanced permeability of aortic tissue in diabetic rats. In addition, sesamin treatment also affected oxidative stress markers; sesamin attenuated the increased MDA content and reduced activity of SOD in diabetic rats.

Vascular dysfunction is a complicating feature of diabetes in humans and experimental models, and hyperglycemia is the primary cause of micro- and macrovascular complications in the diabetic condition (17). Vascular dysfunction and enhanced permeability in the diabetic rat might be due to increased blood glucose levels and decreased blood insulin levels. Hyperglycemia has been shown to cause tissue damage through several mechanisms, including advanced glycation end product (AGE) formation, increased polyol pathway flux, apoptosis, and reactive oxygen species (ROS) formation (18). Our results showed that sesamin treatment did not have a hypoglycemic effect in STZ-induced diabetic rats; therefore, its beneficial effect on the permeability of aortic tissue is likely due to mechanisms other than a hypoglycemic effect. Sesamin has been shown to have an anti-inflammatory property (19), and this may have led to the decreased vascular permeability observed in the diabetic rats in our study. In addition, some of the damaging effects on the vascular tissue of diabetic animals are believed to be due to enhanced oxidative stress, as shown by increased MDA and decreased activity of defensive enzymes like SOD (15), as was observed in this study. This could also lead to diabetes-induced functional changes in vascular endothelial cells and the subsequent development of vascular malfunction. The results of the present study showed that chronic treatment with sesamin significantly decreased MDA content and increased SOD activity in the aortic tissue of diabetic rats, indicating that the improvement in vascular permeability may be due in part to the amelioration of lipid peroxidation and oxidative injury. These results clearly suggest that another possible reason for the effect of sesamin on the improvement of endothelial dysfunction and vascular abnormality is due to its antioxidant capacity. There is also some evidence that nitric oxide depletion is partly responsible for the increased permeability of the vascular system in the diabetic condition (20), and sesamin-induced enhancement of endothelial NOS activity may have reduced the permeability of aortic tissue in diabetic rats3.

In conclusion, to the best of our knowledge, this is the first study reporting that chronic sesamin treatment could dose-dependently improve aortic permeability in diabetic rats, partly through attenuation of oxidative stress. Our data may be helpful in the development of new natural drugs for diabetes that improve endothelial function and prevent cardiovascular diseases.

\section{Acknowledgments}

Authors would like to thank Fariba Ansari for her excellent technical assistance.

\section{Financial Disclosure}

None declared.

\section{Funding/Support}

This study was financially supported by the Iranian National Science Foundation (grant \# 85122/65), which is affiliated with the Presidential Office of Iran.

\section{References}

1. Wild S, Roglic G, Green A, Sicree R, King H. Global prevalence of diabetes: estimates for the year 2000 and projections for 2030. Diabetes Care. 2004;27(5):1047-53.

2. Coccheri S. Approaches to prevention of cardiovascular complications and events in diabetes mellitus. Drugs. 2007;67(7):997-1026.

3. Kong X, Yang JR, Guo LQ, Xiong Y, Wu XQ, Huang K, et al. Sesamin improves endothelial dysfunction in renovascular hypertensive rats fed with a high-fat, high-sucrose diet. Eur J Pharmacol. 2009;620(1-3):84-9.

4. Naito M, Fujikura J, Ebihara K, Miyanaga F, Yokoi H, Kusakabe T, et al. Therapeutic impact of leptin on diabetes, diabetic complications, and longevity in insulin-deficient diabetic mice. Diabetes. 2011;60(9):2265-73.

5. Ikeda S, Kagaya M, Kobayashi K, Tohyama T, Kiso Y, Higuchi N, et al. Dietary sesame lignans decrease lipid peroxidation in rats fed docosahexaenoic acid. J Nutr Sci Vitaminol (Tokyo). 2003;49(4):270-6.

6. Hirose N, Doi F, Ueki T, Akazawa K, Chijiiwa K, Sugano M, et al. Suppressive effect of sesamin against 7,12-dimethylbenz[a]-anthracene induced rat mammary carcinogenesis. Anticancer Res. 1992;12(4):1259-65.

7. Kita S, Matsumura Y, Morimoto S, Akimoto K, Furuya M, Oka N, et al. Antihypertensive effect of sesamin. II. Protection against two-kidney, one-clip renal hypertension and cardiovascular hypertrophy. Biol Pharm Bull. 1995;18(9):1283-5.

8. Nakano D, Itoh C, Ishii F, Kawanishi H, Takaoka M, Kiso Y, et al. Effects of sesamin on aortic oxidative stress and endothelial dysfunction in deoxycorticosterone acetate-salt hypertensive rats. Biol Pharm Bull. 2003;26(12):1701-5.

9. Rogi T, Tomimori N, Ono Y, Kiso Y. The mechanism underlying the synergetic hypocholesterolemic effect of sesamin and alpha-tocopherol in rats fed a high-cholesterol diet. J Pharmacol 
Sci. 2011;115(3):408-16.

10. Yamashita K, Iizuka Y, Imai T, Namiki M. Sesame seed and its lignans produce marked enhancement of vitamin E activity in rats fed a low alpha-tocopherol diet. Lipids. 1995;30(11):1019-28.

11. Nakano D, Kwak CJ, Fujii K, Ikemura K, Satake A, Ohkita M, et al. Sesamin metabolites induce an endothelial nitric oxide-dependent vasorelaxation through their antioxidative property-independent mechanisms: possible involvement of the metabolites in the antihypertensive effect of sesamin. J Pharmacol Exp Ther. 2006;318(1):328-35.

12. Konan AB, Datte JY, Yapo PA. Nitric oxide pathway-mediated relaxant effect of aqueous sesame leaves extract (Sesamum radiatum Schum. \& Thonn.) in the guinea-pig isolated aorta smooth muscle. BMC Complement Altern Med. 2008;8:23.

13. St-Pierre P, Bouffard L, Papirakis ME, Maheux P. Increased extravasation of macromolecules in skeletal muscles of the Zucker rat model. Obesity (Silver Spring). 2006;14(5):787-93.

14. Roghani M, Baluchnejadmojarad T. Chronic epigallocatechingallate improves aortic reactivity of diabetic rats: underlying mechanisms. Vascul Pharmacol. 2009;51(2-3):84-9.

15. Baluchnejadmojarad T, Roghani M. Chronic administration of genistein improves aortic reactivity of streptozotocin-diabetic rats: mode of action. Vascul Pharmacol. 2008;49(1):1-5.

16. Bradford MM. A rapid and sensitive method for the quantitation of microgram quantities of protein utilizing the principle of protein-dye binding. Anal Biochem. 1976;72:248-54.

17. Madonna R, De Caterina R. Cellular and molecular mechanisms of vascular injury in diabetes-part I: pathways of vascular disease in diabetes. Vascul Pharmacol. 2011;54(3-6):68-74.

18. Hartge MM, Unger T, Kintscher U. The endothelium and vascular inflammation in diabetes. Diab Vasc Dis Res. 2007;4(2):84-8.

19. Lee WJ, Ou HC, Wu CM, Lee IT, Lin SY, Lin LY, et al. Sesamin mitigates inflammation and oxidative stress in endothelial cells exposed to oxidized low-density lipoprotein. J Agric Food Chem. 2009;57(23):11406-17.

20. Chakir M, D'Orleans-Juste P, Plante GE. Neutral endopeptidase inhibition, a new approach in the exploration of diabetic vasculopathy in rats. Eur J Pharmacol. 1995;285(1):11-8. 